\title{
9
}

\section{RELATIONSHIP BETWEEN THE COMPOSITION OF SOLID SOLAR-SYSTEM MATTER AND DISTANCE FROM THE SUN}

\author{
J. T. WASSON
}

\begin{abstract}
The chondritic meteorites have "solar" compositions, indicating that they have aroided fractionation in planetary processes such as partial melting or fractional crystallization. Since chondrites contain solar proportions of volatiles having nebular condensation temperatures $5500 \mathrm{~K}$, it follows that agglomeration of grains and accretion to parent bodies occurred after the nebula had cooled to such low temperatures, and, that models calling for simultaneous condensation and accretion of high-temperature minerals such as Fe-Ni metal or ferromagnesian silicates are implausible.

Two independent intergroup fractionations have been recoqnized in chondritic materials; refractory element abundances (e.g., the ca/si ratiol and degree of oxidation measured by the FeO/(FeO + MqO) ratio decrease through the chondrite sequence: carbonaceous-ordinary-IAB-E. Hiatus between groups result from incomplete sampling by the Earth of the original spectrum. A plausible speculation is that this sequence reflects formation at different nebular pressures and temperatures, the fine-grained, oxidized, volatile-rich carbonaceous chondrites forming at lower temperatures $>5 \mathrm{AU}$ from the sun, and the reduced enstatite chrondrites forming at higher temperatures near the sun. The high fall frequency of the three groups of ordinary chondrites suggests an origin in the asteroid belt. The degree of oxidation of the IAB chondrites appears to be slightly lower than that of the Earth, suggesting an origin near or slightly loss than $1 \mathrm{AU}$ from the Sun. The o-isotope compositions of chondrites are consistent with this pirture.

Asteroids tend to be light or dark, with a hiatus in properties between these two classes. This can be understood if the light materials are ordinary chondrites originally formed at 2.2-3.5 AU, whereas the dark materials originated at $>5 A U$, and were deposited in the asteroid belt during a later period as a result of a major increase in the influx of cometary materials through the inner so?ar system associated with the generation of the oort cloud of comets.
\end{abstract}

We know that the composition of the planets differ hecause they have different densities. [ensities of the outer planets are low because they contain large amounts of light, volati Je elements such as $\mathrm{H}$ and He (Jupiter, Saturn) or $\mathrm{C}, \mathrm{N}$ and $O$ (Uranus, Neptune). In contrast, the inner planets have low volatile contents and substantially higher mean densities. Fig. la shows the observed den- 

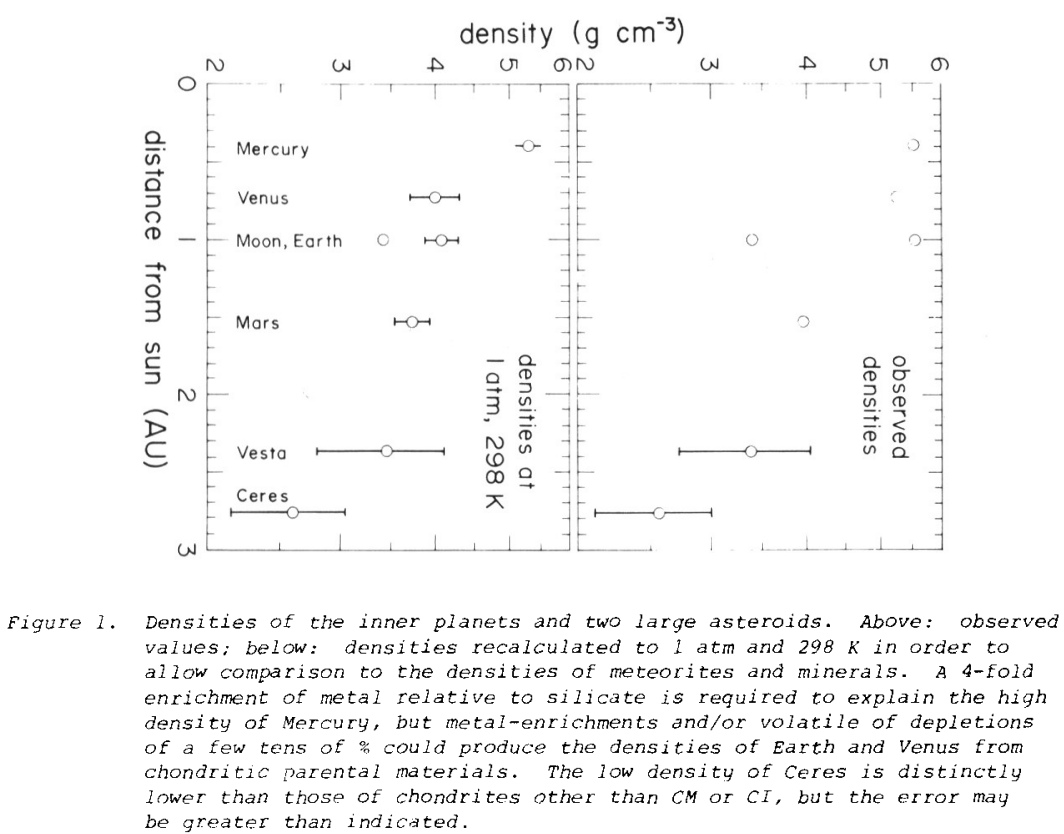

sities of the inner planets together with those of two large asteroids, 1 Ceres and 4 vesta. Fig. Ib shows the densities recalculated to 1 atm and $298 \mathrm{~K}$, a necessary step in order to compare them with the densities of mincral phases (Reynolds and Summers, 1969). Because these calculat ions involve assumptions regarding the compositions of the planetary interiors and uncertainties in equations of state, these calculated densities have large error limits. Note, for cxample, that the reduced densities of Venus and Earth are unresolved. In contrast, uncertainties in the densities of the asteroids chiefly result from uncertainties in the measured masses and radii. With the exception of Mercury and the asteroid Ceres, the calculated densities fall in the range $3.4-4.1 \mathrm{~g} \mathrm{~cm}^{-3}$.

Various pieces of evidence indicate that the chondritic meteorites are primitive and relatively unfractionated samples of non-volatile solar-system matter. Their compositions are quite different from those found in igneous rocks, which generally consist of mineral assemblages having a rather sharp $\left(-50^{\circ}\right)$ range of melting temperatures; chondrites melt over an extended $(22010)$ range. Some members of each chondrite group consist of disequilibrium mineral assemblages that cannot easily be explained in terms of planetary-type igneous processes, but can be accounted for by a combination of equilibrium and kinctic processes occurring in the solar nebula. Especially important is the observation that equilibrium with nebular gases at relatively low $(\leqslant 500 \mathrm{~K})$ temperatures is demanded in order to understand the abundance of highly volatile elements such as In or Cd (Anders, 1971). This indicates that agglomeration of $\mathrm{mm}$ and submm grains occurred only after the nebula cooled to very low temperatures, and casts serious doubt on planetary formation models calling for sequential accretion onto the surfaces of growing planets of phases in the order in which they form in a cooling solar nebula (Turekian and Clark, 1969). It appears that the most plausible models are those that consider chondritic materials to be the hulding hlocks of the planets.

In Table I are listed several properties of the 10 major groups of chondritic meteorites. The classification of the meteorites follows the principles outlined 
Table I. Properties of chondritic meteorites.

\begin{tabular}{|c|c|c|c|c|c|c|}
\hline Group & Clan & $\begin{array}{c}\mathrm{Fe} / \mathrm{Si} \\
(\mathrm{atom} \%)\end{array}$ & $\begin{array}{l}\mathrm{Ca} / \mathrm{Si} \\
(\text { atom } \%)\end{array}$ & $\begin{array}{c}\mathrm{FeO} /(\mathrm{FeO}+\mathrm{MgO}) \\
(\text { mole } \%)\end{array}$ & $\begin{array}{l}\text { Density } \\
\left(g^{\circ ~} \mathrm{~cm}^{-3}\right)\end{array}$ & $\underset{(\%)}{\text { Fall }}$ \\
\hline $\mathrm{CI}^{+}$ & \multirow{4}{*}{$\begin{array}{l}\text { carbo- } \\
\text { naceous } \\
\text { chondrites }\end{array}$} & $86-93$ & $6.9-7.9$ & $\sim 40$ & 2.3 & 0.68 \\
\hline $\mathrm{CH}^{+}$ & & $80-85$ & $6.4-7.0$ & $\sim 35$ & 2.8 & 1.9 \\
\hline $\mathrm{CO}^{+}$ & & $81-85$ & $6.5-7.1$ & 230 & 3.4 & 0.81 \\
\hline $\mathrm{Cl}_{i}^{+}$ & & $76-80$ & $9.1-9.9$ & $\sim 30$ & 3.4 & 1.1 \\
\hline $\mathrm{LL}$ & \multirow{3}{*}{$\begin{array}{l}\text { ordinary } \\
\text { chondrites }\end{array}$} & $50-56$ & $4.5-5.0$ & $27-32$ & 3.5 & 7.2 \\
\hline $\mathrm{L}$ & & $55-62$ & $4.7-5.1$ & $21-25$ & 3.6 & 39.0 \\
\hline $\mathrm{H}$ & & $75-83$ & $4.8-5.2$ & $16-19$ & 3.7 & 32.1 \\
\hline $\operatorname{IAB} 3^{\frac{1}{r}}$ & IAB irons & $50-80$ & $3.9-4.5$ & $4-7$ & 3.8 & 1.0 \\
\hline E6 & \multirow{2}{*}{$\begin{array}{l}\text { enstatite } \\
\text { chondrites }\end{array}$} & $60-70$ & $3.4-3.8$ & $0.1-0.2$ & 3.6 & 0.81 \\
\hline$E 4,5$ & & $90-100$ & $3.3-3.7$ & $0.5-1.0$ & 3.8 & 0.68 \\
\hline
\end{tabular}

* Fraction of meteorites recovered following an observed fall.

+ The groups of carbonaceous chondrites are each named for an important member. The Ivuna-group (CI) chondrites are volatile-rich $\left(20 \% \mathrm{H}_{2} \mathrm{O}\right.$, 43 C) and generally considered to be the most prinitive chondrites; they are often designated type-I carbonaceous chondrites or C1. The Wighei-group (CM) chondrites have slightly lower volatile abundances; nost chondrites designated type-II carbonaceous or C2 are members of this group. The Vigarano-group (CV) and Ornans-group (CO) chondrites have much lower volatile contents (no $\mathrm{H}_{2} \mathrm{O}, \leqslant 1 \%$ C); some researchers combine them to a single group designated type-III carbonaceous or C3 chondrites.

* Chondritic materials present as angular fragments having dimensions $0.1-10 \mathrm{~cm}$ set in a metallic (iron meteorite) matrix.

in Wasson (1974). It is interesting to compare the densities of the chondrites with those of the inner planets, hearing in mind that differentiation processes such as core formation, fractional crystallization of silicate magmas, etc., have relatively little effect on the 1 -atm, $298 \mathrm{~K}$ density of a system having a fixed composition. Several of the less oxidized chondrite groups have densities similar to that of Mars, whereas no chondrites are as dense as the Earth, Venus or Mercury. This suggests either that the selection of chondritic materials in Table $I$ is incomplete and that some having higher densities exist, or that some segregation of higher density from lower density phases occurred during agglomeration and accretion. In fact, there is evidence for both. The chondritic materials in the unique Netschatvo meteorite have a measured density of $4.1 \mathrm{~g} \mathrm{~cm}^{-3}$ (Buchwald, 1975), and it can be shown that removal of a few $\%$ volatile $(\mathrm{H}, \mathrm{C}, \mathrm{N}, \mathrm{Cl}, \mathrm{Na})$ from $\mathrm{E} 4$ chondrites would raise their density to $3.9 \mathrm{~g} \mathrm{~cm}^{-3}$. A density as high as that of Mercury requires a major enrichment of metal relative to other phases. I will return to this point.

Two major types of fractionations can be seen in Table I. Abundances relative to $\mathrm{Si}$ of elemints such as Ca that are refractory during nebular-condensition processes decrease through the chondrite sequence $\mathrm{CV}$-other carbonaccous-ordinary-AB-enstatite. The parameter $\mathrm{FeO} /(\mathrm{FeO}+\mathrm{MgO})$ reflecting the degree of oxidation of the meteoritic matcrial al so decreases through the same sequence, except 
that ratios in the $C V$ and $C o$ chondrites are slightly lower than those in $C M$ and $C I$ carbonaceous chondrites. Current understanding indicates that different nebular processes were responsible for the fractionation of these two parameters. Their narrow ranges within groups and large variations among the chondrites as a whole seem best understood in terms of a very incomplete terrestrial sampling of the original set of primitive nebular materials. It is probable that nebular pressure and temperature decreased monotonically with increasing distance from the Sun. According to Blander and Abdel-Gawad (1969), the higher the nebular pressure the more reduced the condensate. The refractory fractionation probably resulted from incomplete agglomeration of a high-temperature condensate; this process may have depended on pressure, but no quantitative discussion is available. If refractory abundance and degree of oxidation varied systematically with increasing distance from the Sun, these parameters offer valuable information about the formation locations of chondritic meteorites. During the remainder of this paper I examine this admittedly speculative model.

The very small grain size and the high volatile contents (including $20 \% \mathrm{H}_{2} \mathrm{O}$ ) of the $\mathrm{CM}$ and $\mathrm{CI}$ chondrites indicate that these formed at rather low temperatures, as expected in the outer portions of the solar nehula (Anders, 1971). The low degree of oxidation of the $E$ chondrites is most consistent with formation at high temperatures near the Sun. Thus, the data are consistent with the sequence E-IABordinary-carbonaceous forming at increasing distances from the Sun. The Ca/Si and $\mathrm{FeO} /(\mathrm{FeO}+\mathrm{MgO})$ data from Tahle I are illustrated in Fig. 2 with one modification: $\mathrm{E} 4,5$ and $\mathrm{E} 6$ are plotted together at $50.1 \% \mathrm{FeO} /(\mathrm{FeO}+\mathrm{MgO})$ because all the former and the higher values reported for $\mathrm{B}$ (h are out of equilibrium with the metal. More details are given in Wasson (1977).

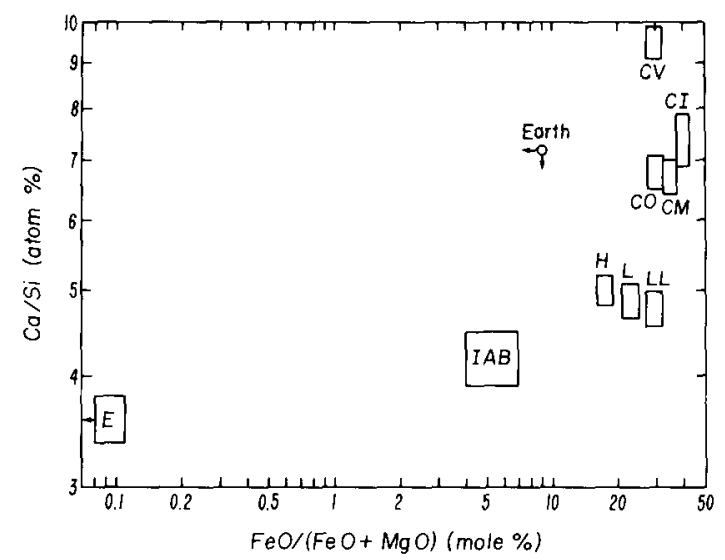

\footnotetext{
Figure 2. Two independent parameters, refractory abundance (represented b; Ca/Si) and degree of oxidation (measured by Feo/ (Feo + MgO)l increasunntis monotonically through the sequence E-IAB-ordinary $(H, L, L L)$-cabonaceous (CI, CM, CO, CV) chondrites. It is speculated that this fractionation sequence reflects formation at increasing distances from the sun.
}

As noted above, a reasonable a priori assumption is that the iuncr planets formed by accretion of materials having chondritic bulk compositions. If we knew the hulk composition of each planet, we could plot these on Fig. 2 as a test of the model and, if it passed the test, as a calibration of the fractionation trend in terms of distance from the Sun. In fact, we know very little about the bulk compositions of planets other than the Earth, and our knowledge of the Earth's composition is only approximate. The point labelled Earth on Fig. 2 is the com- 
position Ringwood (1970) estimates for pristine upper mantle material. This must be considered an upper limit on both parameters, since differentiation of the mantle must have increased the $\mathrm{Ca}$ abundance in the upper mantle, and late accretion of chondritic material (after formation of the core and the crust) will have increased the $\mathrm{FeO} /(\mathrm{FeO}+\mathrm{MgO})$ ratio in the outer parts of the Earth. The latter should be a relatively small effect, however, and it seems safe to conclude that the degree of oxidation of the Earth is similar to or slightly greater than that of the more oxidized members of group IAB. Because of the large hiatus in FeO/ $(\mathrm{FeO}+\mathrm{MgO})$ between $\mathrm{IAB}$ and $\mathrm{E}$ materials, it seems likely that the latter formed $\ll 1$ AU from the Sun, perhaps at a location intermediate between the orbits of venus and Mercury.

Table I shows that the three groups of ordinary chondrites are the most common meteorites among observed falls. Correction for destruction during deceleration in the Earth's atmosphere would raise the frequency of the $\mathrm{CI}$ and, to a lesser extent, the $C M$ chondrites, but the other chondrite groups are comparable to the ordinary chondrites in tensile strength, and their fall frequencies reflect genuinely low abundances in Earth-crossing orbits. The most plausible explanation of the high abundance of the three ordinary chondrite groups is that they formed in and still account for most of the mass of the asteroid belt, the largest source of debris inside the orbit of Jupiter. Note that if the sequence in Fig. 2 is indeed one of increasing distance from the Sun, an origin of the ordinary chondrites in comets (Wetherill, 1968) formed at $>5$ All from the sun would require IAB or F group chondrites or a similar but st ill rarer form of chondrite to be associated with the asteroid helt.

Spectral studies of asteroids (Chapman, 1976; Morrison, 1976; Ze1lner and Bowell, 1976) show that the surfaces of most asteroids fall in one or the other of two categories: C-type having low albedos $(0.02-0.06)$ and rather foatureless spectra, or S-type having higher albedos $(0.08-0.25)$ and evidence for electronic transitions in the ir spectra. The hiatus in spectral properties (especially alhedo) is surprising, since it seems unlikely that the properties of chondritic materials formed in this relatively small (2.2 - 3.4 Alu portion of the solar system would show such a wide range of propertics, and still less likely that two groups separated by a distinct hiatus in properties would be formed.

It appears to me that the simplest way to account for these two distinct types of materials is that one formed in the asteroid helt, whereas the other formed elsewhere in the solar system, and was transported into the belt at a later time. Whipple (1976) noted that a second period of aceretion mily have occurred in the inner solar system $10^{7}-10^{8}$ years after the formation of the terrestrial planets as a result of a high influx of cometary material resulting from the perturhations necded to move comets from the major planet region into the oort cloud. These cometary solid materials could reaccumulate as deposits on existing asteroids. The amount of material accumulated could be quite substantial; Whipple suggests that the amount of solid matter in the inner solar system might have been greater than $10^{25} \mathrm{~g}$, the present mass of the asteroid belt. Thus asteroidal deposits of outer-solar system material lo or more $k m$ deep are not implausible. Impacts of such parent bodies will tend to either he crater-producing. which would leave the asteroid in the ( spectral class, or fragmenting, which would lead to a dominant exposure of decper lying materials helonging to the high-albedo spectral classes.

In summary, available data on chondrites and asteroids suggest the following

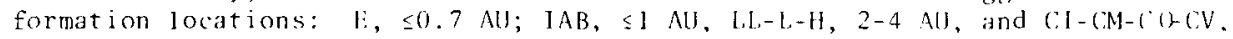
35 All. One or more of the C classes may exist as deposits on asteroids; others may have comets as the ir parent bodies.

The best way to test these ideas would he to send sample-recovery spacecraft to comets and asteroids, with asteroids showing evidence of fragmentation (c.g., irregular shapesf being the more interesting targets. less-direct tests now available are provided by the spectrum of Mercury and 0-isotope data. 
My scenario jmplies that the hulk silicate composition Mercury should be chondritic, but highly reduced, and that the $\mathrm{FeO} /(\mathrm{FeO}+\mathrm{MgO})$ ratio in the ferromagnesian minerals in the surface rocks should be quite low. Were it not for contamination by late-acereted chondritic material the ratios should be as low as those in $\mathrm{E}$ chondrites. Vilas and Mecord 19976$)$ reported several visible re-

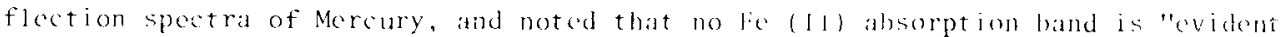
to the precision of the data," consistent with this view. However, lunar studies show that weathering processes tend to reduce the magnitude of the $0.9 \mu \mathrm{m}$ band, and confirmation of the low feo content must await high-resolution studies of fresh Mercurian craters.
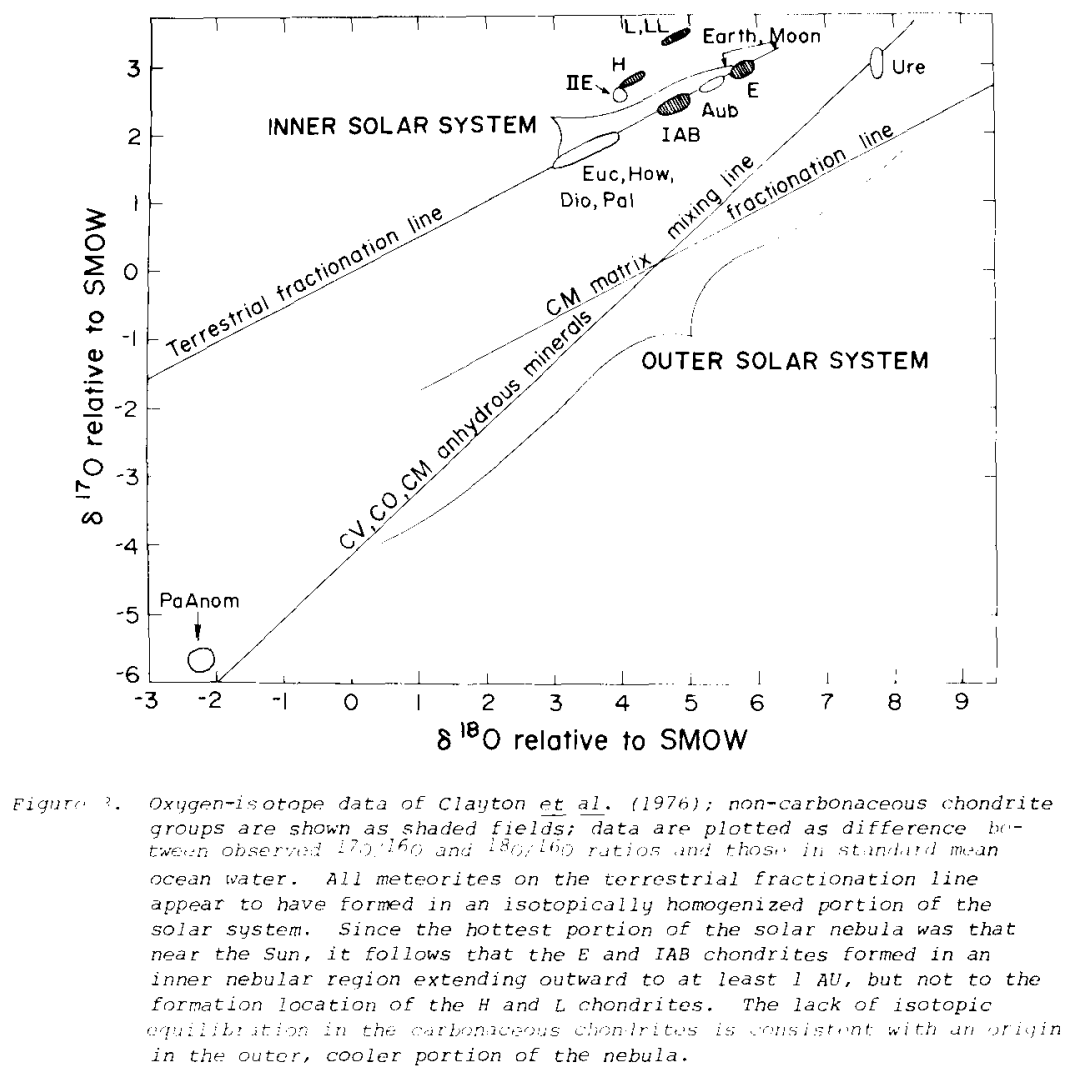

A summary of the $O-$ isotope data for major meteorite classes is shown in Fig. 3 (Clayton et al., 1976). Non-carbonaceous chondrites are shown als shaded fjelds. Two of these, the IA and E fall along the "terrestrial fractionation line" with slope 0.5 which results from small amounts of chemical or physical fractionation of material having the same composition as terrestrial ocean waters. All studied terrestrial samples lie along this line. Bulk analyses of carbonaccous chondrites are not available, but high-temperature minerals fall along a "(CV, CO, CM mixing line" that cannot be produced by fractionation, hut scems to result from mixing of end components lying outside the range shown in Fig. 3. Clayton has suggested that the $160-r i c h$ end component results from interstellar solids. The CM matrix fractionation line shows that the low temperature material in (M chondrites has 


\section{COMPOSITION AND DISTANCE}

experjenced fractionation processes, but was never in equilibrium with matter of terrestrial 0-isotope composition. The lack of equilibration of carbonaceous chondrites indicates that all phases could not have formed hy sequential condensation of an initially well-mixed gas. This is consistent with their formation in the conler, outer portion of the solar nebula. The hot test portion of the solar nebula was that nearest the Sun. That the E and IAP chondrites lie along the terrestrial fractionation line indicates that they formed in the inner solar system, and also that the solar system was well mixed out to $1 \mathrm{AU}$. That the ordinary chondrites plot off the terrestrial fractionation line indicates that they formed outside the well mixed inner portion of the nebula. lf they formed in the asterojd belt, the outer limit of the jsotopically well-mixed portion of the nehula did not extend past 2 All.

The high abundance of chondrites among meteorites and the presence in chondrites of solar abundances of elements with condensation temperatures as low as $500 \mathrm{~K}$ suggests that the chondrites are the building blocks of the plinets. This idea has recently been out of favor partly bocause of the high densitics of Mercury, Venus and the Earth. However, the 1 atm, $298 \mathrm{~K}$ densities of Venus and Earth can he understood in terms of a molest increase in metal relative to silicate, possibly augmented by some loss of volatiles. The densities of Mercury requires an $\mathrm{Fe} / \mathrm{Si}$ ratio $4 \mathrm{X}$ higher than that in $\mathrm{E} 4,5$ chondrites, but the fact that such fractionations did occur in the solar nebula is indicated by the observations (1) that the E4,5 Fe/Si ratio is $2 X$ higher than that in E6 chondrites, and (2) that very high $(>10) \mathrm{Fe} / \mathrm{Si}$ ratios were produced in IAB meteorites by nebular processes (Wasson, 1970; Scott, 1972).

\section{ACKNOWLEDGEMENTS}

The discussion in this paper includes improvements resulting from conversations with numerous individuals, but especially $W$. V. Boynton and G. W. Wetherill. This research is mainly supported by NSF grant EAR 74-22495.

\section{REFERENCES}

Anders, E. 1971, Ann. Rev. Astron. Astrophys., 9, 1.

Blander, M., and Ahdel-(iawad, M. 1969, Geochim. Cosmochim. Acta, 33, 701.

Buchwald, V. F. 1975, in Handbook of Iron Meteorites, Univ. of Calif. Press, $1418 \mathrm{pp}$.

Chapman, C. R. 1976, IAU Collog. 39, this volume.

Clayton, R. N., Omuma, N., and Mayeda, T. K. 1976, Earth Planet. Sci. Lett., 30, 10 .

Morrison, D. 1976, IAU COllog. 39, this volume.

Reynolds, R. 'l', and Summers, A. L. 1969, J. Geophys. Rev., 74, 2494.

Ringwood, A. E. 1970, Phys. Earth Planet. Interiors, 3, 109.

Scott, E.R.D. 1972, Geochim. Cosmochim. Acta, 36, 1205.

Turekian, K. K., and Clark, S. P. 1969, Earth Planet. Sci. Lett, 6, 346.

Vilas, R., and Mccord, T. B. 1976, Icarus, 28, 593.

Wasson, .J. T. 1970, Icarus, 12, 407.

Wasson, J. T. 1974, Meteorites--Classification and Properties, springer, 316 pp. Wasson, J. 'T. 1977, Formation Locations of Chondritic Meteorites, in preparation. Wetheri11, G. W. 1968, Science, 159, 79.

Whipple, F. L. 1976, Mem. Soc. Roy. Sci. (Liege), Ser. 6, 9, 101.

Zellner, B., and Bowell, E. 1976, IAU Collog. 39, this volume. 
LIPSCHUTZ: By what mechanism do you propose that enstatite chondrites were. brought into the asteroidal belt from near the orbit of Mercury?

WASSON: If the parent bodies formed at a location intermediate between Mercury and Venus, perturbations by Venus could place a small fraction into Mars crossing orbits, and Mars perturbations could decouple some of these from Venus and Earth. The dynamic lifetime of material in Mars-crossing orbits is about $10^{9}$ yr, long enough to allow a few to survive until the present. The enstatite chondrites account for only $1.5 \%$ of observed falls, thus a strong source is not required.

ANDERS: It is unlikely that carbonaceous chondrites come from distances greater than $5 \mathrm{AJ}$. As $I$ showed in a paper in Icarus (1974), most carbonaceous chondrites contain solar-wind derived noble gases in such high concentrations that they must have formed less than 4-5 AU from the sun. This was later confirmed by Price's group at Berkeley who derived a similar limit from solar flare tracks in these meteorites.

WASSON: This may indeed represent a serious constraint. I will look into the evidence you mention.

CHAPMAN: You have suggested that the ordinary chondrites formed primarily in the asteroidal region and that the several carbonaceous types formed at greater distances from the sun. Although you appeal to unspecified celestial mechanical processes to move materials around the solar system and perhaps deposit bodies formed elsewhere into the asteroid belt, your picture is still difficult to reconcile with the known distribution of asteroid spectral types. We find that well over $80 \%$ of the asteroids of $2100 \mathrm{~km}$ diameter are of apparent carbonaceous composition and ordinary-chondrite-like asteroids are present but very rare in the main belt. It is often suggested that, since we are making astronomical observations of asteroid surfaces we do not know that asteroidinteriors are carboraceous. But the asteroid diameters are now known to be so large that simple collisional calculations demonstrate that asteroids are nearly all frigments, many of them relatively recent fragments. Thus present asteroid surfaccs are quite probably representative of hulk properties of precursors. In the present collisional environment of the belt (tupical relative velocities $\sim 5 \mathrm{~m} / \mathrm{sec}$ ) it is difficult to envision any process that could coat ordinary chondritic asteroids with a carbonaceous coating; rather there is a rapid net loss of asteroid surface material. Thus it is difficult to imagine that the bulk of main belt asteroids car be ordinary chondritic composition.

WASSON: There are serious problems with reconciling the presence of asteroids having spectra indicating differentiated materials (Vesta) or ordinary-chondritelike materials, (Dembowska) with the observation that the spectra of most asteroids are best matched by laboratory of CI or CM chondrites that chiefly consist of hydrated silicates. What possible conditions cculd have lead to the production of materials having no hydrated silicates in precisely the same region as where CI or CM-like materials formed? It seems there is no way to plausibly account for these observations by known condensation or agglomeration process. Therefore, we are forced to conclude that one or the other of these materials formed elsewhere and were subsequently transported to the asteroid belt. It appears more likely to me that the low-albedo, featureless spectra either reflect coatings of cometary materials deposited on the asteroids or, alternatively, of darkening of the surfaces by glass formation, a very important process on the Moon. I would Iike to see an attempt at a quantitative treatment of these possibilities.

WETHERILL: I think it would be useful to distinguish between the model-dependent 
and model-independent aspects of your work. At the present time we have no satisfactory theory of the formation of the sun, and do not know if the planets and meteorite parent bodies formed before or after the sun; the classical angular momentum problem remajns unsolved etc. Therefore, conclusions specifying distances from the sun and relationships to the orbits of particular planets which are implicitly model-dependent have a much greater probability of ultimately being proven incorrect; on the other hand, features which are relatively model-independent could survive shifting fashions in theories of solar system formation. I also think it would be helpful if model-dependent conclusions were developed quantitatively and prefaced by a brief statement indicating the general class of model employed, rather than leaving this implicit.

WASSON: I agree with your remarks. However, my hypothesis is essentially independent of nebular models. I have reported an empirjcal correlation between two seemingly independent parameters, and proposed that the observed trend may reflect formation at monotonically increasing distances from the sun. The hypothesis appears to be independent of model-dependent explanations that might be used to explain the variations in each of these parameters.

WOOD: A general comment: It is worth noting that the preceding papers have been cast largely in terms of the "Cameron nebula," a model of solar system formation wherein a relatively rapid collapse of interstellar gas and dust causes compression and adiabatic heating of the inner solar system nebula, at least out to the present asteroid belt, which vaporizes the interstellar dust grains. The system then cools, and metallic elements recondense; it is the fine structure of this recondensation that has been under discussion. Now Dr. Cameron has reconsidered his model, and no longer believes the solar nebula was substantially heated farther out from the present orbit of Mercury. To the best of my knowlsdge, no other astrophysicist has championed the concept of a hot nebula. Thus, the condensation models under discussion currently have no theoretical underpinning.

WASSON: Geochronological date indicate that the chondrites were formed in the solar nebula. The mineralogy of enstatite, ordinary, $\mathrm{CV}$ and $\mathrm{CO}$ chondrites, and the chemical fractionations such as the refractory element/si ratios observed between these groups demands that temperatures in the inner portion of the solar nebula have reached $1500-2000 \mathrm{~K}$. Theoretical nebular models which do not yield such high temperatures are incorrect or incomplete. 